\title{
INFLUENCE OF NORDIC WALKING TRAINING ON MUSCLE STRENGTH AND THE ELECTROMYOGRAPHIC ACTIVITY OF THE LOWER BODY IN WOMEN WITH LOW BONE MASS
}

\author{
ZBIGNIEW OSSOWSKI ${ }^{1}$, EWELINA LIEDTKE², KATARZYNA SIEMIENIUK², SANDRA \\ SZYDEOWSKA $^{3}$, PIOTR SAKOWSKI ${ }^{3}$, WOJCIECH SKROBOT ${ }^{4}$ \\ ${ }^{I}$ Gdansk University of Physical Education and Sport, Faculty of Tourism and Recreation, \\ Department of Health Promotion \\ ${ }^{2}$ PhD Studies, Gdansk University of Physical Education and Sport, Faculty of Physical Education \\ ${ }^{3}$ Master's Studies, Gdansk University of Physical Education and Sport \\ ${ }^{4}$ Gdansk University of Physical Education and Sport, Faculty of Rehabilitation and Kinesiology, \\ Department of Kinesiology
}

Mailing address: Zbigniew Ossowski, Gdansk University of Physical Education and Sport, 1 Kazimierza Górskiego
Street, 80-336 Gdańsk, tel.: +48 505113037, fax: +48 58 3418460, e-mail: zbigniew.ossowski@awf.gda.pl

\begin{abstract}
Introduction. Osteoporosis and osteopenia are related to changes in the quantity and quality of skeletal muscle and contribute to a decreased level of muscle strength. The purpose of this study was to evaluate the impact of Nordic walking training on muscle strength and the electromyographic (EMG) activity of the lower body in women with low bone mass. Material and methods. The participants of the study were 27 women with low bone mass. The sample was randomly divided into two groups: a control group and an experimental group. Women from the experimental group participated in 12 weeks of regular Nordic walking training. Functional strength was assessed with a 30-second chair stand test. The EMG activities of the gluteus maximus (GMax), rectus femoris (RF), biceps femoris (BF), soleus (SOL), and lumbar (LB) muscles were measured using a surface electromyogram. Results. Nordic walking training induced a significant increase in the functional strength $(\mathrm{p}=0.006)$ of the lower body and activity of GMax $(\mathrm{p}=0.013)$ and a decrease in body mass $(\mathrm{p}=0.006)$ in women with reduced bone mass. There was no statistically significant increase in the EMG activities of the RF, BF, SOL, or LB muscles. The study did not indicate any significant changes in functional muscle strength, the EMG activity of the lower body, or anthropometry in women from the control group. Conclusions. Nordic walking training induces positive changes in lower body strength and the electromyographic activity of the gluteus maximus as well as a decrease in body mass in women with low bone mass.
\end{abstract}

Key words: women, low bone mass, functional strength, EMG activity, Nordic walking

\section{Introduction}

Nordic walking (NW) has become very popular nowadays. A recent study found that 10 million people participate in this sporting activity in various countries [1]. Existing research mainly focuses on presenting it as beneficial for the elderly [2]. Hagner et al. state that a 12-week moderate intensity Nordic walking programme can cause significant decreases in BMI, total fat mass, LDL, triglycerides, and waist circumference as well as an increase in HDL in premenopausal and perimenopausal women [3]. Other studies suggest that marching with poles is effective in improving the physical fitness of elderly people $[4,5]$. Moreover, it exerts beneficial effects on resting heart rate, blood pressure, exercise capacity, maximal oxygen consumption, and quality of life in patients with various diseases and can thus be recommended to a wide range of people as primary and secondary prevention [6]. It was also revealed that NW has a favourable influence on the strength of the lower limbs in Systolic Hypertensive Postmenopausal Women [7]. However, in the literature on this subject, we have not found any research that would dem- onstrate the influence of Nordic walking training on muscle strength and the electromyographic activity of the lower body in women with low bone mass. The problem of strength loss in women with osteoporosis is especially crucial in the light of recent studies which show that postmenopausal women with osteopenia have 17.6-21\% lower quadriceps strength than healthy control subjects [8]. Higher lean mass and muscle strength were positively associated with BMDs [9]. Sarcopenia, on the other hand, is associated with low BMD and osteoporosis. Subjects with sarcopenia were twice as likely to have osteoporosis as normal subjects [9]. According to the findings of other studies, osteoporosis in connection with the postmenopausal period can negatively affect the growth of leg strength in physically active women to a significant extent. Brench et al. report that women with postmenopausal osteoporosis presented diminished knee extensor and flexor muscle strength compared to women without postmenopausal osteoporosis [10].

Leg muscle strength is important because it affects the limits on the performance of daily activities. One of the important basic tasks of daily living is rising from a chair, a type of motion 
which requires important joint torques, coordination, and accurate balance control. With ageing, the ability to rise from a chair and sit down tends to deteriorate, which constitutes a major source of disability and handicap [11]. This raises the risk of falling, causes the elderly to be increasingly dependent on others in daily living activities, and can lead to institutionalisation [12]. Therefore, it was decided that the best procedure to make an assessment of muscle strength was to apply a functional test, specifically the 30-second chair stand test, which is recommended for planning and organising rehabilitation for elderly people [13].

The purpose of the study was to evaluate the impact of NW training on functional muscle strength and the electromyographic activity of the lower body in women with low bone mass.

\section{Material and methods}

Twenty-seven subjects aged 65-75 (68.52 \pm 4.79$)$ years were included in the study. The participants were women with low bone mass defined by a T-score $\leq-1$ [14]. All the participants were recruited from the 740 women who agreed to take part in the primary screening and attended a variety of lectures at the University of the Third Age. The sample was randomly divided into two groups. The first group was a control group (CG) consisting of 14 women $(68.57 \pm 4.7$ years $)$. Participants in the control group were involved in normal daily activities according to their age range. None of the subjects were engaged in an organised physical activity regimen. Women from the second group, called the experimental group (EG), participated in regular Nordic walking training ( $\mathrm{n}=13 ; 68.46 \pm 5.08$ years). Subjects were excluded from the study according to the following criteria: uncontrolled hypertension, oophorectomy, rheumatoid arthritis, pulmonary disease, or type 2 diabetes treated with insulin.

All of the participants recruited for this study had to undergo a compulsory medical examination which revealed no contraindications to physical exercise.

This experiment was approved by the Bioethics Committee of the Regional Medical Chamber.

\section{Measures}

The participants were tested two times - prior to the beginning of the training programme (baseline) and after three months of training (post-training).

\section{Assessment of anthropometry}

Body weight was measured with octopolar bioimpedance (InBody 720, Biospace, Seoul, Korea), using standard protocols with participants wearing light clothing and without shoes.

Body height was measured to an accuracy of $0.1 \mathrm{~cm}$. During the measurement, the subject was placed barefoot in the orthostatic position. The body mass index was calculated as body mass divided by height ${ }^{2}\left(\mathrm{~kg} / \mathrm{m}^{2}\right)$.

\section{Assessment of strength and surface EMG}

Functional lower body strength was assessed using a 30-second chair stand test from the Senior Fitness Test battery [15]. The chair stand test measured the number (repetitions) of full stands that were completed in 30 seconds with the arms folded across the chest. The starting position before each trial was standardised by adjusting the foot placement and chair height.

During the 30-second chair stand test, women had bipolar self-adhesive electrodes put on the chosen muscles of the lower body. The electrodes registered muscle activity during muscle contraction, which made it possible to measure surface EMG using a TELEmyo Direct Transmission System (Noraxon U.S.A. Inc.). EMG data were collected using the software application MyoResearch_XP Master Edition. The electrodes were placed on the skin over the bellies of the gluteus maximus (GMax), rectus femoris (RF), biceps femoris (BF), soleus (SOL), and lumbar (LB) muscles.

Electromyographic data were synchronised with a cinematic system to define the beginning and end of each 30-second chair stand test. Cinematic data were recorded with a digital camera (Sony NEX- FS700R) and analysed using the MyoResearch_XP Master Edition system.

\section{Training programme}

All the exercise sessions, each lasting $60 \mathrm{~min}$, were held outdoors, three times a week for 12 weeks. Each training unit consisted of three phases. During the warm-up phase (10-13 $\mathrm{min}$ ), the women performed exercises with poles. The main part of the Nordic walking training $(40 \mathrm{~min})$ consisted in walking a distance of 3-4 $\mathrm{km}$ with poles, with an average heart rate of between $40-60 \%$ of an age-dependent maximal heart rate calculated using the following formula: (HRmax - HRrest) $\mathrm{x}(0.4$ to 0.6$)+$ HRrest; HRmax $=206-0.88 \times$ age [16]. The cool down exercises (7-10 $\mathrm{min}$ ) included dynamic and static stretching. The heart rate was monitored using a Polar RS-400 heart-rate monitor (Polar Electro Oy, Finland).

\section{Statistical analysis}

Standard statistical methods were used to calculate means and standard deviations (mean \pm standard deviation). The Shapiro-Wilk test was used to check the normality of the data. The paired t-test or the Wilcoxon test was used for within-group comparisons. Between-group comparisons of percentage difference changes were performed using the Mann-Whitney $U$ test. In addition, effect size was calculated to describe the magnitude of a treatment effect.

All data were analysed using the statistical package Statistica 10 (StatSoft, 2010). The level of significance was set at $\mathrm{p}<0.05$.

\section{Results}

The anthropometric data and results recorded for muscle strength and electromyographic activity in the subjects with low bone mass are shown in table 1.

At baseline, no statistically significant differences were observed between the EG and CG in the parameters analysed. However, within-group analysis performed after 3 months showed a significant improvement in lower limb strength $(p=0.006)$ in the EG. In the CG, the change in the mean results obtained in the 30-second chair stand test was not statistically significant.

The changes in the average results for lower limb strength in subjects from the CG and EG are presented in figures 1 and 2.

Tests also revealed a significant increase in the electromyographic activity of the gluteus maximus $(\mathrm{p}=0.01)$ in subjects taking part in NW training. However, after 3 months, a higher dispersion of EMG results was noted (especially in the EG). Table 1 presents the values of the standard deviations, which support this observation. Additionally, there was no statistical improvement in the activity of the muscles analysed in women from the CG. The mean changes in the EMG activity of the lower body of the participants are presented in figures 3 and 4 . 
Table 1. Results obtained for anthropometry, muscle strength, and the electromyographic activity of the lower limbs for all the subjects

\begin{tabular}{|c|c|c|c|c|c|c|c|c|c|}
\hline \multirow{3}{*}{ Variables } & \multicolumn{3}{|c|}{ Baseline (month 0) } & \multicolumn{3}{|c|}{ Month 3} & \multicolumn{2}{|c|}{ Change $\%$} & \multirow{3}{*}{ ES } \\
\hline & EG & \multirow{2}{*}{$P$} & CG & $E G$ & \multirow{2}{*}{$P$} & CG & EG & CG & \\
\hline & Mean \pm SD & & Mean \pm SD & Mean $\pm S D$ & & Mean \pm SD & Mean $^{1} \pm S D^{1}$ & Mean $^{2} \pm S D^{2}$ & \\
\hline \multicolumn{10}{|l|}{ Anthropometry } \\
\hline $\mathrm{BM}(\mathrm{kg})$ & $67.55 \pm 9.28$ & & $66.91 \pm 9.88$ & $66.69 \pm 9.38$ & & $67.65 \pm 9.48$ & $-1.27 \pm 1.08^{*}$ & $1.11 \pm 4.05$ & -0.80 \\
\hline BMI $\left(\mathrm{kg} / \mathrm{m}^{2}\right)$ & $26.4 \pm 4.14$ & & $25.98 \pm 4.32$ & $26.36 \pm 4.28$ & & $26.15 \pm 4.27$ & $-0.15 \pm 3.38$ & $0.65 \pm 1.16$ & -0.32 \\
\hline CST (rep) & $16.68 \pm 2.71$ & & $16.18 \pm 2.77$ & $17.9 \pm 3.45$ & & $16.47 \pm 2.85$ & $7.31 \pm 27.3^{*}$ & $1.79 \pm 2.89$ & 0.28 \\
\hline \multicolumn{10}{|l|}{$E M G(\mu \mathrm{V})$} \\
\hline GMax & $6.09 \pm 4.12$ & & $4.7 \pm 2.43$ & $15.69 \pm 25.8$ & & $5.88 \pm 4.06$ & $157.6 \pm 526^{*}$ & $25.1 \pm 67$ & 0.35 \\
\hline RF & $15.49 \pm 6.95$ & & $16.56 \pm 8.9$ & $23.28 \pm 32.21$ & & $15.58 \pm 6.56$ & $50.3 \pm 363.5$ & $-5.92 \pm 26.8$ & 0.22 \\
\hline $\mathrm{BF}$ & $8.79 \pm 4.28$ & & $12.85 \pm 7.5$ & $12.33 \pm 10.73$ & & $9.11 \pm 3.96$ & $40.27 \pm 150.7$ & $-29.1 \pm 47.5$ & 0.62 \\
\hline SOL & $12.87 \pm 6.06$ & & $13.47 \pm 6.42$ & $17.02 \pm 16.27$ & & $13.37 \pm 5.6$ & $32.25 \pm 168.5$ & $-0.74 \pm 11.5$ & 0.28 \\
\hline LB & $18.32 \pm 7.27$ & & $17.12 \pm 7.4$ & $28.73 \pm 29.37$ & & $18.03 \pm 6.5$ & $56.82 \pm 304$ & $5.32 \pm 11.8$ & 0.24 \\
\hline
\end{tabular}

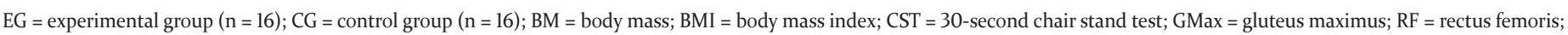
$\mathrm{BF}=$ biceps femoris; $\mathrm{SOL}=$ soleus; $\mathrm{LB}=$ lumbar; change $\%=[($ after 3 months - baseline $) /$ baseline $] \times 100 ;$ effect size $(\mathrm{ES})=\left(\mathrm{mean}^{1}-\mathrm{mean}^{2}\right) /\left[\right.$ root mean square $\left.\left(\mathrm{SD}^{1}+\mathrm{SD}^{2} / 2\right)\right]$;

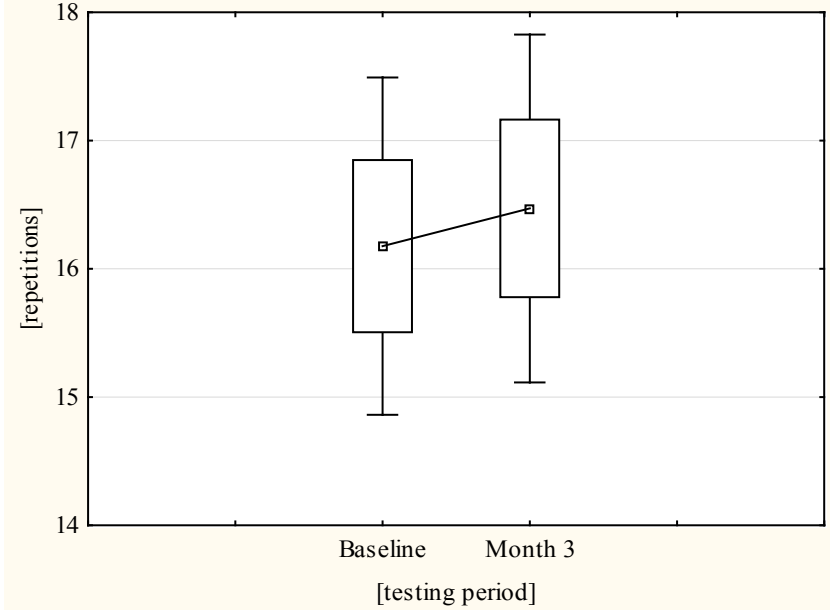

Figure 1. Changes in average results obtained in the 30-second chair stand test for CG subjects

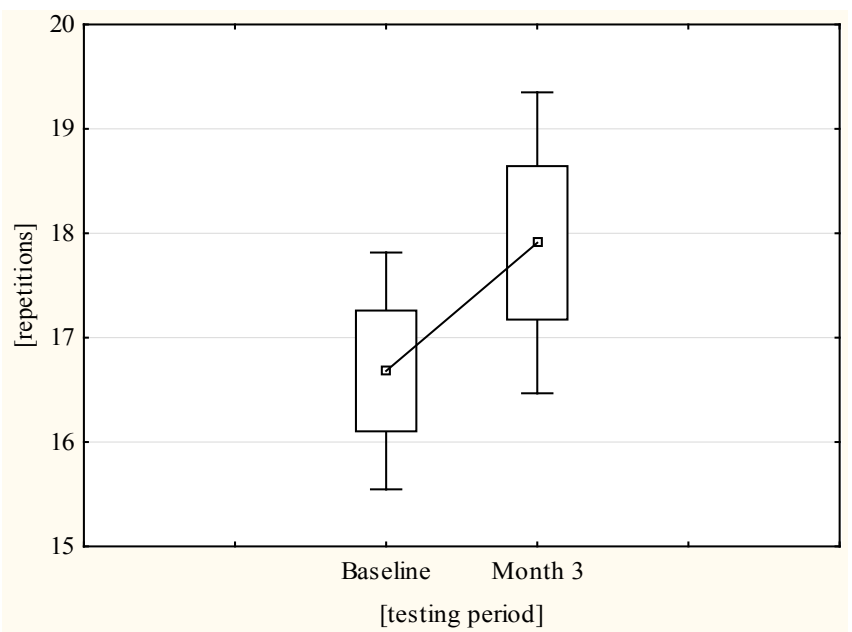

Figure 2. Changes in average results obtained in the 30-second chair stand test for EG subjects

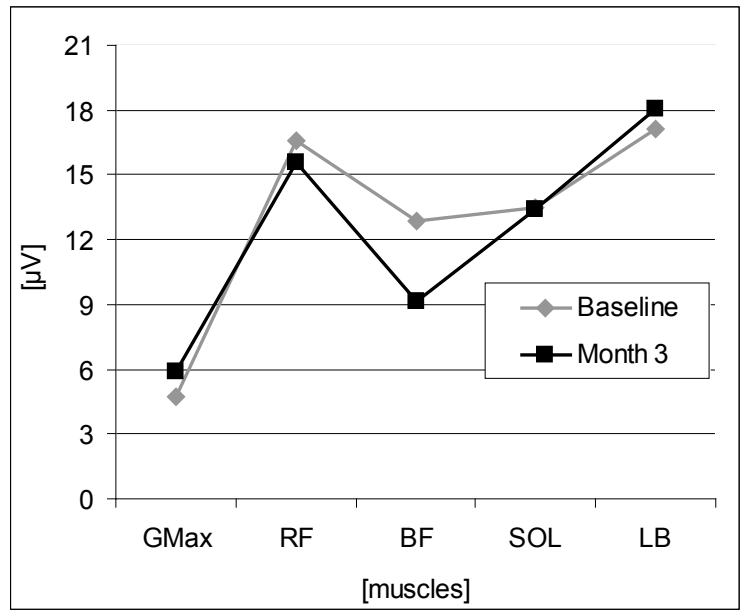

Figure 3. Changes in average results of surface EMG in CG subjects

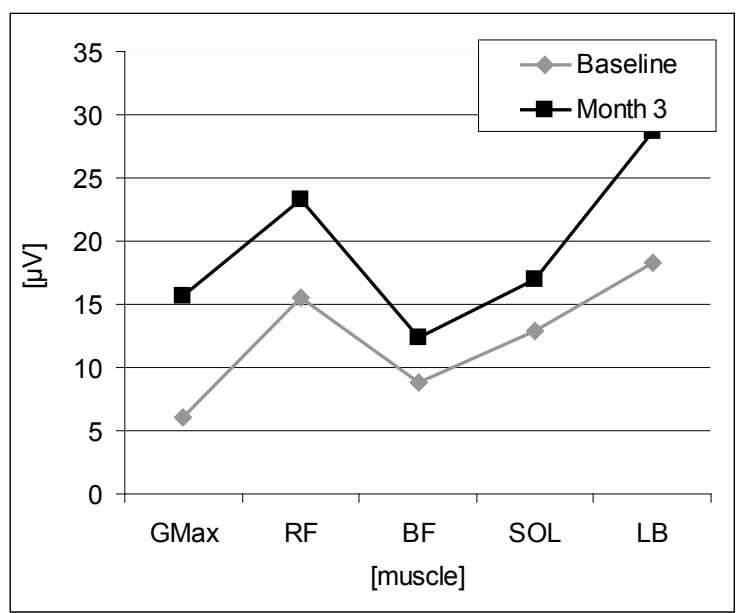

Figure 4. Changes in average results of surface EMG in EG subjects 
Moreover, Nordic walking training significantly decreased BM ( $\mathrm{p}=0.006)$ and BMI $(\mathrm{p}=0.05)$ in women with low bone mass. Conversely, no significant change in muscle strength, electromyographic activity, or anthropometry was observed.

After 3 months, differences in the analysed variables between the EG and CG were not statistically significant.

\section{Discussion}

Changes in muscle strength and the electromyographic activity of the lower limbs are pronounced during the ageing process [17]. Such changes are especially dangerous for women with low bone mass [18].

Amaral et al. claim that the lower limbs are more affected by ageing than the upper limbs, as demonstrated by a greater reduction in isometric muscle strength, fat-free mass, and EMG signal power. They suggest that differences between the upper and lower limbs are related to neuromuscular adaptations derived from greater activation of the upper limbs, as compared to the lower limbs. Therefore, exercises requiring more frequent activation of lower-limb muscles may be beneficial [17].

The results obtained during the 30 -second chair stand test showed that mean muscle strength rose by $7.31 \%$ as a result of NW, which was a statistically significant change.

A similar tendency was observed in other studies. Kocur et al. described the effects of Nordic walking on functional fitness in cardiac patients. These authors also found improvements in muscle strength after Nordic walking, but not after conventional walking [19]. This research demonstrates that greater strength benefits can be derived from Nordic walking, as compared to conventional walking. However, when comparing the strength benefits of Nordic walking to resistance training, the improvements in arm curl and chair stand observed for Nordic walking were smaller than those observed for resistance training alone [20]. Therefore, although Nordic walking provides benefits to muscular strength, if one is to maximise improvements in muscular strength, resistance training should be employed.

Hughes et al. estimate that elderly subjects use up to $97 \%$ of the strength of their knee extensors when rising from a low chair [21]. Even if the knee extensor group is often considered as the main actor in the sit-to-stand (STS) movement, Gross et al. state that the hip extensors (gluteus maximus) play a role which is even more important than that of the knee extensors, in particular in the vertical acceleration phase [22]. The present study has also demonstrated a positive, though not statistically significant, improvement of the electromyographic activity of the rectus femoris (50.3\%), biceps femoris (40.27\%), soleus (32.25\%), and lumbar (56.82\%) muscles in women from the EG.

The study conducted by us did not reveal any significant change in functional muscle strength or the electromyographic activity of the lower body in women from the CG.

To our knowledge, there is a lack of research showing the impact of Nordic walking training on the electromyographic activity of the lower body in women with low bone mass, which resulted in difficulties with comparing our data with those obtained by others. However, Sugiyama et al. state that the iEMG readings for the lower extremities were significantly lower during NW than during level walking in healthy adults who regularly engaged in physical activity in their daily lives. The authors suggest that the use of poles in NW attenuates the muscle activity of the lower extremities during the stance and push-off phases [23]. Similar results were obtained by other researchers who assessed the effect of Nordic pole walking on the electro- myographic activities of upper and lower extremity muscles in adults (21.38 \pm 0.87 years) [24]. These authors observed that the average and maximum values for the muscle activity of the quadriceps, hamstring, tibialis anterior, and gastrocnemius muscles in the lower extremities did not show large differences in either group (walking with and without Nordic poles), and the values did not show any statistically significant differences between the groups. In conclusion, the researchers suggested that the use of Nordic poles increased the muscle activity of the upper extremities compared with regular walking but did not affect the lower extremities.

It is highly probable that the lack of statistically significant improvement in the activity of the rectus femoris, biceps femoris, soleus, and lumbar muscles in the NW group is related to the above noted observation.

On the other hand, our study has demonstrated that NW exerts beneficial effects on the activity of the gluteus maximus $(157.6 \%)$ in women with reduced bone mass. To interpret the above result, it is worth paying attention first to studies on the effects of Nordic walking and walking on gait parameters.

According to Shim (2012), when compared with walking without Nordic poles, walking using Nordic poles increased step length and the time from heel contact to foot flat while reducing the time from foot flat to heel off [25]. Similar results were also obtained by other researchers, who reported that the Nordic walking group showed an increase in cadence, stride length, and step length, in comparison with the walking group [26].

It is possible that longer step during Nordic walking activates hip extension muscles (especially the gluteus maximus) more than other muscles in the lower extremities, which could explain the results observed. Because of the lack of studies showing the impact of Nordic walking training on the electromyographic activity of the gluteus maximus, this is only a hypothesis that must be verified in the next phase of research.

The results of the current study are important because the muscular strength of the lower extremities is a major factor affecting the stability of balance vs. falling [27]. Moreover, Nordic walking increases the stride and can be considered helpful for patients with diseases affecting gait [26].

Further analysis showed that the training undergone by the subjects had a statistically significant influence on decreasing BM $(-1.27 \%)$, as opposed to the findings for the non-active women from the CG. Our results confirm the findings presented by South Korean scientists [28], who examined changes in body composition in three groups (Nordic walking, normal walking, and control) after the two walking groups practised walking 3 times a week, for 60 minutes, during a 12-week period. In this study, there was a significant difference in the weight of the Nordic walking group before and after the training period, which amounted to $-1.67 \%$.

In recent years, there have been several publications on Nordic walking and its effect on human health [29]. The present study extends our knowledge on the positive impact of NW training on functional muscle strength and the EMG activity of the lower body in women with low bone mass.

\section{Conclusions}

The current study proves that 12 weeks of NW training are efficient in increasing lower body strength and the electromyographic activity of the gluteus maximus as well as in decreasing body mass in women with low bone mass. These results suggest that Nordic walking training may in part contribute to clinically 
relevant improvements in muscle activities and functional lower body strength in patients with osteopenia and osteoporosis.

\section{Acknowledgements}

The study was conducted under the EU project "Active lifestyles and predictors of risk for incapacity on senior population - RISINC2013”.

\section{Literature}

1. International Nordic Walking Federation. (2011). INWA Nordic Walking Portal. Retrieved from http://www.inwanordicwalking.com.

2. Piech K., Piech J., Grants J. (2014). Nordic walking - a versatile physical activity fit for everyone (a literature review). LASE Journal of Sport Science 5(1), 48-58.

3. Hagner W., Hagner-Derengowska M., Wiacek M., Zubrzycki I.Z. (2009). Changes in level of VO2max, blood lipids, and waist circumference in the response to moderate endurance training as a function of ovarian aging. Menopause 16(5), 1009-13. DOI: 10.1097/gme.0b013e31819c0924.

4. Sokeliene V., Cesnaitiene V.J. (2011). The influence of Nordic walking on physical fitness of elderly people. Education Physical Training Sport 3(82), 45-51.

5. Ossowski Z., Prusik K., Prusik K., Kortas J., Wiech M., Bielawa Ł. (2014). Nordic walking training and physical fitness in elderly women. Polish Journal of Sport and Tourism 21(4), 253-257. DOI: 10.1515/pjst-2015-0006.

6. Tschentscher M., Niederseer D., Niebauer J. (2013). Health benefits of Nordic walking: a systematic review. American Journal of Preventive Medicine 44(1), 76-84. DOI: 10.1016/j. amepre.2012.09.043.

7. Latosik E., Zubrzycki I.Z., Ossowski Z., Bojke O., Clarke A., Wiacek M. et al. (2014). Physiological responses associated with Nordic walking training in systolic hypertensive postmenopausal women. Journal of Human Kinetics 43, 185190. DOI: 10.2478/hukin-2014-0104.

8. Hakestad K.A., Nordsletten L., Torstveit M.K., Risberg M.A. (2014). Postmenopausal women with osteopenia and a healed wrist fracture have reduced physical function and quality of life compared to a matched, healthy control group with no fracture. BMC Women's Health 3, 14-92. DOI: 10.1186/1472-6874-14-92.

9. He H., Liu Y., Tian Q., Papasian C.J., Hu T., Deng H.W. (2016). Relationship of sarcopenia and body composition with osteoporosis. Osteoporosis International 27(2), 47382. DOI: 10.1007/s00198-015-3241-8.

10. Brech G.C., Alonso A.C., Luna N.M., Greve J.M. (2013). Correlation of postural balance and knee muscle strength in the sit-to-stand test among women with and without postmenopausal osteoporosis. Osteoporosis International 24(7), 2007-13. DOI: 10.1007/s00198-013-2285-x.

11. Dehail P., Bestaven E., Muller F., Mallet A., Robert B., Bourdel-Marchasson I. et al. (2007). Kinematic and electromyographic analysis of rising from a chair during a "Sitto-Walk" task in elderly subjects: role of strength. Clinical Biomechanics (Bristol, Avon) 22(10), 1096-103.

12. Guralnik J.M., Winograd C.H. (1994). Physical performance measures in the assessment of older persons. Aging (Milano) 6(5), 303-5.
13. Różańska-Kirschke J., Kocur P., Wilk M., Dylewicz P. (2006). The Fullerton Fitness Test as an index of fitness in the elderly. Medical Rehabilitation 10(2), 9-16.

14. Nelson H.D., Helfand M., Woolf S.H., Allan J.D. (2002). Screening for postmenopausal osteoporosis: a review of the evidence for the U.S. Preventive Services Task Force. Annals of Internal Medicine 137(6), 529-41.

15. Rikli R.E., Jones C.J. (1999). Development and validation of a functional fitness test for community-residing older adults. Journal of Aging and Physical Activity 7, 129-161. DOI: 10.1093/geront/gns071.

16. Gulati M., Shaw L.J., Thisted R.A., Black H.R., Bairey Merz C.N., Arnsdorf M.F. (2010). Heart rate response to exercise stress testing in asymptomatic women: The St. James Women Take Heart Project. Circulation 122, 130-7. DOI: 10.1161/ CIRCULATIONAHA.110.939249.

17. Amaral J.F., Alvim F.C., Castro E.A., Doimo L.A., Silva M.V., Novo Júnior J.M. (2014). Influence of aging on isometric muscle strength, fat-free mass and electromyographic signal power of the upper and lower limbs in women. Brazilian Journal of Physical Therapy 18(2), 183-90.

18. Edwards M.H., Dennison E.M., Aihie Sayer A., Fielding R., Cooper C. (2015). Osteoporosis and sarcopenia in older age. Bone 80, 126-30. DOI: 10.1016/j.bone.2015.04.016.

19. Kocur P., Deskur-Smielecka E., Wilk M., Dylewicz P. (2009). Effects of Nordic walking training on exercise capacity and fitness in men participating in early, short-term inpatient cardiac rehabilitation after an acute coronary syndrome - a controlled trial. Clinical Rehabilitation 23, 995-1004. DOI: 10.1177/0269215509337464.

20. Takeshima N., Islam M.M., Rogers M.E., Rogers N.L., Sengoku N., Koizumi D. et al. (2013). Effects of Nordic walking compared to conventional walking and band-based resistance exercise on fitness in older adults. Journal of Sports Science and Medicine 12(3), 422-430.

21. Hughes M.A., Myers B.S., Schenkman M.L. (1996). The role of strength in rising from a chair in the functionally impaired elderly. Journal of Biomechanics 29(12), 1509-13.

22. Gross M.M., Stevenson P.J., Charette S.L., Pyka G., Marcus R. (1998). Effect of muscle strength and movement speed on the biomechanics of rising from a chair in healthy elderly and young women. Gait and Posture 8(3), 175-185.

23. Sugiyama K., Kawamura M., Tomita H., Katamoto S. (2013). Oxygen uptake, heart rate, perceived exertion, and integrated electromyogram of the lower and upper extremities during level and Nordic walking on a treadmill. Journal of Physiological Anthropology 32(1), 2. DOI: 10.1186/18806805-32-2.

24. Shim J.M., Kwon H.Y., Kim H.R., Kim B.I., Jung J.H. (2013). Comparison of the effects of walking with and without Nordic pole on upper extremity and lower extremity muscle activation. Journal of Physical Therapy Science 25(12), 1553-6. DOI: $10.1589 /$ jpts.25.1553.

25. Shim J.M. (2012). Comparison of gait and feet during Nordic pole walking and unassisted walking on a treadmill. Journal of Physical Therapy Science 24, 1225-1228.

26. Park S.K., Yang D.J., Kang Y.H., Kim J.H., Uhm Y.H., Lee Y.S. (2015). Effects of Nordic walking and walking on spatiotemporal gait parameters and ground reaction force. Journal of Physical Therapy Science 27(9), 2891-3. DOI: 10.1589/ jpts.27.2891.

27. Aoyama M., Suzuki Y., Onishi J., Kuzuya M. (2011). Physical and functional factors in activities of daily living that predict falls in community-dwelling older women. Geri- 
atrics and Gerontology International 11, 348-357. DOI: 10.1111/j.1447-0594.2010.00685.x.

28. Song M.S., Yoo Y.K., Choi C.H., Kim N.C. (2013). Effects of Nordic walking on body composition, muscle strength, and lipid profile in elderly women. Asian Nursing Research (Korean Society of Nursing Science) 7, 1-7. DOI: 10.1016/j. anr.2012.11.001.
29. Piech K., Raczyńska B. (2010). Nordic walking - a versatile physical activity. Polish Journal of Sport and Tourism 17, 6978.

Submitted: April 25, 2016

Accepted: June 13, 2016 